\title{
Voice-Related Quality of Life (V-RQOL) Following Type I Thyroplasty for Unilateral Vocal Fold Paralysis
}

\author{
$* \dagger$ Norman D. Hogikyan, $\$$ Walter P. Wodchis, *§Jeffrey E. Terrell, \\ *Carol R. Bradford, and IIRamon M. Esclamado \\ *Department of Otolaryngology-Head and Neck Surgery, University of Michigan Health System; †Vocal Health Center, \\ University of Michigan Health System; $\neq$ School of Public Health, University of Michigan, Ann Arbor, Michigan; \\ $\$ H e a l t h$ Services and Development Center for Excellence, Veterans Affairs Hospital, Ann Arbor, Michigan; \\ IFormerly affiliated with Department of Otolaryngology-Head and Neck Surgery, University of Michigan \\ Health System, Ann Arbor, Michigan: currently affiliated with Department of Otolaryngology- \\ Head and Neck Surgery, The Cleveland Clinic Foundation, Cleveland, Ohio
}

\begin{abstract}
Summary: Unilateral vocal fold paralysis is a common clinical problem which frequently causes severe dysphonia. Various treatment options exist for this condition, with the type I thyroplasty being one of the more commonly performed surgical procedures for vocal rehabilitation. The Voice-Related Quality of Life (VRQOL) Measure is a validated outcomes instrument for voice disorders. This study measured the V-RQOL of patients with unilateral vocal fold paralysis who had undergone a type I thyroplasty and compared these scores to those of patients with untreated and uncompensated unilateral vocal fold paralysis and to normals. Treated patients had significantly higher domain and overall V-RQOL scores than untreated patients, but also scored lower than normals. These differences were true across gender and age. Patients who were more distant from surgery had lower VRQOL scores than those who had more recently been treated. It is concluded that type I thyroplasty leads to a significantly higher V-RQOL for patients with unilateral vocal fold paralysis. This study also demonstrates further the utility of patientoriented measures of treatment outcome. Key Words: Unilateral vocal fold paralysis-Voice-related quality of life (V-RQOL)-Type I thyroplasty.
\end{abstract}

Unilateral vocal fold paralysis (UVFP) usually causes severe dysphonia and can also lead to dys-

Accepted for publication July $30,1999$.

This paper was presented at The Voice Foundation's 28th Annual Symposium: Care of the Professional Voice, June 6, 1999, Philadelphia, Pennsylvania,.

Address correspondence and reprint requests to Norman D. Hogikyan, Department of Otolaryngology, University of Michigan Medical Center, 1904 Taubman Center, 1500 East Medical Center Drive, Ann Arbor, Michigan 48109-0312, USA. e-mail: nhogikya@umich.edu phagia. Treatment options for UVFP are varied, and were recently reviewed in an article by the principal author. ${ }^{1}$ Laryngeal framework surgery consisting of the type I thyroplasty (TPY I) is one of the more commonly employed surgical treatment options for patients with persistent dysphonia due to UVFP. This procedure medializes the paralyzed vocal fold to enhance glottic closure and vibratory dynamics. The surgical literature contains several papers describing procedures considered to be precursors of the contemporary operation, 2,3 but Isshiki et al is credited with description of the fundamental procedure used 
today 4,5 and popularized in the United States by Koufman and others. ${ }^{6}$

Numerous authors have examined treatment outcomes following TPY I using perceptual, acoustic, aerodynamic, and videostroboscopic assessments of voice and of laryngeal function. ${ }^{7-14}$ It is uniformly felt that this procedure can improve and in some cases eliminate the dysphonia associated with UVFP as measured by such methods. Noting the importance of patient-oriented measures of outcome, Harries and Morrison wrote that "the degree of patient satisfaction is one of the most important parameters of the success of surgical treatment, perhaps more than any objective changes."11 Two recent papers ${ }^{15,16}$ explored issues of quality of life (QOL) as related specifically to vocal fold paralysis, and a third paper17 introduced an instrument to measure QOL issues related to voice disorders in general, or voice-related quality of life (V-RQOL). Baba and colleagues used a nonvalidated survey to demonstrate a negative impact of persistent recurrent laryngeal nerve injury upon the QOL of patients undergoing esophagectomy with regional lymphadenectomy for treatment of esophageal carcinoma. ${ }^{15}$ Gliklich and colleagues recently validated a voice outcome survey (VOS) specifically for the purpose of determining treatment outcome of patients with UVFP. 16 This 5-question instrument contains items related to both voice and swallowing, and was shown to be valid, reliable, and responsive. The authors felt that the brevity of the instrument made it easy to administer, but also made it less comprehensive or diagnostically discriminating than a lengthier questionnaire.

The Voice-Related Quality of Life (V-RQOL) Measure is a two-domain, 10-item instrument that has been shown in a diverse population of dysphonic patients to be valid, reliable, and responsive to change (Appendix A). It is now used clinically to determine the degree of impact that a voice disorder is having upon a patient's V-RQOL, and to measure treatment outcomes in voice patients. The purpose of the current study was to measure the V-RQOL of patients treated for UVFP with a TPY I during a recent 5-year period, and to compare these values with those of subsequent new patients with untreated and uncompensated UVFP, and nonvoice patients with no voicerelated complaints. This study was undertaken with the approval of the University of Michigan Institutional Review Board for Human Studies.

\section{MATERIALS AND METHODS}

\section{Patient groups}

All patients treated surgically for UVFP through the University of Michigan Health System during the period from July 1, 1993 through August 31, 1998 were identified through a search of the institutional surgical database. Patients who had undergone only an injection laryngoplasty using Gelfoam (Pharmacia and Upjohn, Kalamazoo, Michigan) paste, a substance intended to provide temporary voice improvement, were then excluded from the search results. Seventy-three patients who had undergone 77 procedures were identified. These surgical procedures were: 57 TPY I, 4 revision TPY I, 10 laryngeal reinnervation operations, and 6 injection laryngoplasties.

The 61 total TPY I had been performed in 58 patients, and by 4 different surgeons. Of these 58 patients: 9 were dead, 2 had previously undergone implant removal for complications without replacement, 1 has a revision pending, 1 had developed cognitive deficits precluding questionnaire completion, and 1 had recovered mobility in the treated vocal fold. These 14 patients were ineligible for the study, leaving 44 eligible patients in this group. The V-RQOL Measure was mailed to these patients via United States mail with a cover letter explaining the purpose of the study, and asking them to return the questionnaire in a provided self-addressed and stamped envelope. Each patient was also asked to give a rating of his or her current voice using a 5-point Likert-style scale of global voice quality.

Twenty consecutive new patients with UVFP who were seen by the senior author from July 1, 1998 through February 28, 1999, but had not yet undergone surgical treatment by August 31, 1998 were also reviewed. To enhance the comparability of this group with the preoperative status of the treated group, patients with well compensated UVFP who were not candidates for a future TPY I at our institution were then eliminated from the list. We define a paralysis as well compensated when complete glottic closure is seen with phonation during stroboscopy, the speaking voice is perceptually normal to mildly dysphonic, and the patient experiences little to no vocal difficulties with their habitual voice use. Three patients met these criteria, leaving 17 dysphonic patients with UVFP constituting the untreated group. 
These patients had presented for care during the time period when the V-RQOL Measure had already been introduced into routine clinical use, and thus the VRQOL and global voice rating data were obtained from patient charts.

Archival V-RQOL data for 22 nonvoice patients were used for the third group in the present study. These 22 patients had no voice complaints, and their scores are used for purposes of comparisons with a normal or nonvoice-disorder population. They are heretofore referred to as the normal group or normals.

\section{Statistical analyses}

Survey responses from the returned questionnaires were recorded. V-RQOL responses from normals, treated, and untreated patients were merged into a single database. An indicator variable was used to distinguish between patient groups. SAS v6.12 and Microsoft Excel were used to manage the data and calculate results, including statistical tests.

The etiologies for UVFP were grouped into four categories: idiopathic, postsurgical (nonmalignant), malignancy, and other. Frequencies were calculated for demographic characteristics and by etiology. The V-RQOL overall scores were calculated along with Social-Emotional and Physical Functioning domain scores. These scores are based on 10, 4, and 6 items, respectively. The formula used to calculate all three scores (Appendix B) provides an index of V-RQOL on a scale of $0-100$, where 0 is extremely poor $\mathrm{V}$ RQOL and 100 is the highest possible V-RQOL that one could expect to attain.

Missing data were imputed using the mean response for the relevant item based on responses of other patients in the same study population. Sensitivity analyses showed this to have an equivalent effect to removing the item from the individual's scoring formula (data not shown). The only item that required imputation asked about employment related V-RQOL. Because some patients were retired, they left this item blank.

Mean V-RQOL scores, standard deviations, and the range of scores were calculated for patients in each of the three populations. Standard $t$ tests were used to test whether scores from the three populations were similar. Most comparisons were conducted for responses of treated and untreated patients. Reported $P$ values are based on two-tailed significance tests. An $F$ test determined that the sample variance for normals were significantly different from treated patients $(F=1.00, P=0.00$ ), while sample variance from treated and untreated patient populations were not significantly different $(F=0.85, P=$ $0.64)$. As a result, $t$ tests for treated and untreated patients assume equal variances and tests for treated versus normals assume unequal variances. $P$ values from tests are reported in the accompanying tables.

\section{RESULTS}

Thirty of the 44 eligible treated patients returned questionnaires for a response rate of $68 \%$. Demographic data for the three study populations, and etiology of paralysis for the treated and untreated groups are shown in Table 1.

Table 2 shows the means and ranges of domain and overall V-RQOL scores by study group. Scores for the treated group were significantly higher than for

TABLE 1. Demographic Data for All Groups, and Etiology of Paralysis for Treated and Untreated Groups

\begin{tabular}{llll}
\hline & Normals & Untreated & Treated \\
\hline $\mathrm{n}$ & 22 & 17 & 30 \\
Mean age & 47.64 & 52.56 & 61.26 \\
Female & $13(59 \%)$ & $8(47 \%)$ & $14(47 \%)$ \\
Diagnosis category & & & \\
$\quad$ Idiopathic & - & $5(29 \%)$ & $3(10 \%)$ \\
Postsurgical (nonmalignant) & - & $6(35 \%)$ & $19(63 \%)$ \\
Malignancy & - & $4(24 \%)$ & $7(23 \%)$ \\
Other & - & $2(12 \%)$ & $1(3 \%)$ \\
\hline
\end{tabular}


TABLE 2. Means and Ranges for Domain and Overall V-RQOL Scores by Study Group

\begin{tabular}{|c|c|c|c|c|c|c|}
\hline & \multicolumn{2}{|c|}{ Normals } & \multicolumn{2}{|c|}{ Untreated } & \multicolumn{2}{|c|}{ Treated } \\
\hline & Mean $(n=22)$ & (SD) & Mean $(n=17)$ & (SD) & Mean* $(\mathbf{n}=30)$ & (SD) \\
\hline $\begin{array}{l}\text { Social-emotional } \\
\text { (range) }\end{array}$ & $\begin{array}{c}98.86 \\
(81.25,100)\end{array}$ & $(4.16)$ & $\begin{array}{c}39.71 \\
(0.00,87.50)\end{array}$ & (28.64) & $\begin{array}{c}75.00 \\
(25.00,100)\end{array}$ & (24.18) \\
\hline $\begin{array}{l}\text { Physical functioning } \\
\text { (range) }\end{array}$ & $\begin{array}{c}97.35 \\
(85.00,100)\end{array}$ & (5.38) & $\begin{array}{c}27.85 \\
(0.00,66.67)\end{array}$ & $(20.52)$ & $\begin{array}{c}72.70 \\
(37.50,100)\end{array}$ & (18.18) \\
\hline $\begin{array}{l}\text { Overall V-RQOL } \\
\text { (range) }\end{array}$ & $\begin{array}{c}97.95 \\
(75.00,100)\end{array}$ & $(3.71)$ & $\begin{array}{c}32.59 \\
(0.00,75.00)\end{array}$ & (21.38) & $\begin{array}{c}73.62 \\
(35.00,100)\end{array}$ & (19.50) \\
\hline
\end{tabular}

*All $P$-values < 0.01 (treated vs untreated, and treated vs normals).

Abbreviations: SD, standard deviation.

the untreated group, and were also significantly lower than the scores for normals. Categorical self-ratings of global voice quality and mean overall $\mathrm{V}$ RQOL scores by voice rating category are shown in Table 3 for the treated and untreated groups. Most of the untreated patients rated their voices as poor, while very good was the most common rating in the treated group. Relative magnitude of V-RQOL scores correlated well with voice rating category.

In Table 4, mean overall V-RQOL scores by gender and age category are shown for each study group. Treated scores were significantly different from untreated scores within each category. Mean V-RQOL scores versus time since surgery are shown in Table 5 for the treated group. Patients who were over 2 years from surgery had significantly lower scores than patients within 1 year of their TPY I.

TABLE 3. Self-ratings of Global Voice Quality and Overall V-RQOL Scores by Voice Rating for Treated and Untreated Groups

\begin{tabular}{lrrrrr}
\hline & \multicolumn{2}{c}{ Untreated } & & \multicolumn{2}{c}{ Treated } \\
\cline { 2 - 3 } \cline { 5 - 6 } Voice Rating & $\mathbf{n}$ & V-RQOL & & $\mathbf{n}$ & V-RQOL \\
\hline Poor & 13 & 28.01 & & \\
Fair & 4 & 47.50 & & 8 & 57.80 \\
Good & & & & 10 & 63.87 \\
Very good & & & & 11 & 92.50 \\
Excellent & & & & 1 & 90.00 \\
\hline
\end{tabular}

\section{DISCUSSION}

Results of this study clearly demonstrate that the average V-RQOL was much higher in treated versus untreated patients. This was true for the social-emotional and physical functioning domains, as well as for the overall scores. Magnitude of this difference was convincing, with treated scores approximately double the untreated scores, and the level of significance was also impressive at $<0.01$. These unequivocal findings accomplish the primary goal of this study, which was to determine whether such a difference existed or not. They also contribute an important new dimension of support for use of TPY I in the treatment of UVFP.

Because the V-RQOL Measure is a relatively new instrument, preoperative data were not available for the treated group. There were however data for a set of dysphonic patients with untreated and uncompensated UVFP readily available from the pool of new patients presenting for care to the same institution in more recent months. We feel that the vocal capabilities and V-RQOL of the untreated group can be appropriately considered representative of the presumed preoperative status of the treated group.

The self-ratings of global voice quality also showed a different pattern in the treated versus the untreated groups (Table 3), with poor being the most common rating in untreated patients and very good the most common in the treated group. Overall V-RQOL scores correlated well with global voice ratings.

To try to better understand the range in V-RQOL scores demonstrated by the untreated and treated 
TABLE 4. Overall V-RQOL Scores by Gender and Age for Each Study Group

\begin{tabular}{|c|c|c|c|c|c|c|}
\hline & \multicolumn{2}{|c|}{ Normals } & \multicolumn{2}{|c|}{ Untreated } & \multicolumn{2}{|c|}{ Treated } \\
\hline & Mean & (SD) & Mean & (SD) & Mean* & (SD) \\
\hline Female & 96.67 & $(5.45)$ & 27.66 & (24.66) & 67.14 & (20.28) \\
\hline Male & 98.85 & $(2.19)$ & 36.98 & (18.33) & 79.29 & $(17.46)$ \\
\hline \multicolumn{7}{|l|}{ Age (years) } \\
\hline Under 45 & 99.06 & (1.29) & 29.58 & $(27.54)$ & 80.74 & (18.62) \\
\hline $45-64$ & 95.63 & $(5.79)$ & 30.30 & (16.69) & 77.95 & (17.77) \\
\hline $65+$ & 99.58 & $(1.02)$ & 38.96 & (21.44) & $* * 67.67$ & $(20.60)$ \\
\hline
\end{tabular}

*All $P$-values $<0.01$ (treated vs untreated), except $* *<0.05$.

TABLE 5. Domain and Overall V-RQOL Scores by Time Since Surgery for Treated Group

\begin{tabular}{|c|c|c|c|c|c|c|}
\hline & \multicolumn{2}{|c|}{$<1$ Year } & \multicolumn{2}{|c|}{ 1-2 Years } & \multicolumn{2}{|c|}{ Over 2 Years } \\
\hline & Mean $(n=8)$ & (SD) & $\operatorname{Mean}(n=6)$ & (SD) & Mean* $(n=16)$ & (SD) \\
\hline Social-emotional & 85.94 & $(15.86)$ & 80.21 & $(28.05)$ & 67.58 & (22.67) \\
\hline Physical functioning & 84.90 & (13.17) & 75.69 & $(17.91)$ & 65.48 & (16.19) \\
\hline Overall V-RQOL & 85.31 & $(13.66)$ & 77.50 & $(21.75)$ & 66.32 & $(17.06)$ \\
\hline
\end{tabular}

* Significantly different from $<1$ year $(P<0.05)$.

groups, the groups were broken down by gender and age categories (Table 4). A statistically significant difference remained between the two groups though, looking across the gender and age categories. There was a tendency within the treated group for male patients to score higher than females, and for older patients to score lower than younger ones; however, the standard deviations were fairly large and differences were not significant. Differences among the various etiologies for UVFP were also looked at (data not shown); however, the relatively small numbers in some groups precluded meaningful comparisons.

We found the data for the treated group regarding V-RQOL score and time from surgery particularly interesting, although in many ways it probably raises more questions than it answers. The patients who were $>2$ years postsurgery had a significantly lower V-RQOL than patients who were $<1$ year from surgery, but all remained higher than untreated scores. $\mathrm{Lu}$ et al looked at longitudinal changes in voice following TPY I, ${ }^{14}$ and found that perceptual, videostroboscopic, acoustic, and aerodynamic measures remained fairly stable over a 6 -month postoperative period. Longitudinal data over a longer time period are not available from the current literature. It is possible that there truly is a decline in postoperative vocal capabilities over a period of years; continued denervation atrophy or fibrosis in vocal fold musculature could lead to further decline in glottic closure or vibratory dynamics. An alternative explanation may be that vocal capabilities remain constant, but patients' expectations become higher with time and they are more bothered by vocal problems. We have found that patients who have fairly recently been operated upon will think of their voices in comparison with the untreated paralysis voice, while those who are much further from the time of surgery tend to think of their vocal capabilities in comparison with normals. The issue of change over time will also be answered by the ongoing prospective study at our institution exploring a wide variety of vocal outcome parameters longitudinally in patients treated surgically for UVFP.

Further comment is warranted by the fact that mean V-RQOL scores in the treated group were significantly less than those of normals. The relative difference in scores was not as great as for treated ver- 
sus untreated patients, but the difference did exist. This concurs with the clinical observation that treated patients generally feel that their voices perform well in most circumstances, but will not be optimal in times when high vocal intensity or endurance is required. This should not really be surprising when considering the fact that the TPY I is a static, geometric solution for a dynamic neurological problem of laryngeal function. These data speak to the fact that although this is an extremely good treatment option, there is still research to be done in treatment of UVFP. The ideal solution, ability to restore motion-specific innervation to the paralyzed vocal fold, is not yet at hand.

This paper also contributes to the growing interest in measuring patient-oriented outcomes for patients with voice disorders, and demonstrates further the utility of such data. As noted earlier by Harries and Morrison, "the degree of patient satisfaction is one of the most important parameters of the success of surgical treatment."11 Using the V-RQOL Measure or other validated patient-oriented outcomes instru- ment, information at the core of patient satisfaction can be reliably measured.

\section{CONCLUSIONS}

In this study, the average V-RQOL scores of patients with UVFP who had been treated with a TPY I were compared to those of untreated patients and to normals. Treated patients scored significantly higher than untreated patients, but were also lower than normals. The V-RQOL scores of patients who were more distant from surgery were lower than those who had been operated upon more recently. Further research is necessary to better define longitudinal V-RQOL and voice quality changes in patients undergoing TPY I. Patient-oriented variables such as V-RQOL are valuable ways of measuring treatment outcomes.

Acknowledgment: The authors wish to acknowledge Dr Jack Wheeler and the University of Michigan Health Services Research Initiative for technical assistance and financial support related to this project. 


\section{APPENDIX A}

\section{Voice-Related Quality of Life (V-RQOL) Measure}

University of Michigan

NAME: DATE:

We are trying to learn more about how a voice problem can interfere with your day-to-day activities. On this paper, you will find a list of possible voice-related problems. Please answer all questions based upon what your voice has been like over the past two weeks. There are no "right" or "wrong" answers.

Considering both how severe the problem is when you get it, and how frequently it happens, please rate each item below on how "bad" it is (that is, the amount of each problem that you have). Use the following scale for rating the amount of the problem:

$$
\begin{aligned}
& 1=\text { None, not a problem } \\
& 2=\text { A small amount } \\
& 3=\text { A moderate (medium) amount } \\
& 4=\text { A lot } \\
& 5=\text { Problem is as "bad as it can be" }
\end{aligned}
$$

\section{Because of my voice,}

1. I have trouble speaking loudly or being heard in noisy situations.

2. I run out of air and need to take frequent breaths when talking.

3. I sometimes do not know what will come out when I begin speaking.

4. I am sometimes anxious or frustrated (because of my voice).

5. I sometimes get depressed (because of my voice).

6. I have trouble using the telephone (because of my voice).

7. I have trouble doing my job or practicing my profession (because of my voice).

8. I avoid going out socially (because of my voice).

9. I have to repeat myself to be understood.

10. I have become less outgoing (because of my voice).

The overall quality of my voice during the last 2 weeks has been (please circle):
Poor
Fair
Good
Very Good
Excellent 


\section{APPENDIX B}

\section{Scoring Algorithm for V-RQOL Measure}

V-RQOL General Scoring Algorithm

$$
100-\left(\frac{\text { Raw Score }-\# \text { items in domain or total }}{\text { Highest Possible Raw Score }-\# \text { items }}\right) \times 100
$$

Social-Emotional Domain (Items 4, 5, 8, 10)

$$
100-\left(\frac{\text { Raw Score }-4}{16}\right) \times 100
$$

Physical Functioning Domain (Items 1, 2, 3, 6, 7, 9)

$$
100-\left(\frac{\text { Raw Score }-6}{24}\right) \times 100
$$

Total Score (Items 1-10)

$$
100-\left(\frac{\text { Raw Score }-10}{40}\right) \times 100
$$

Example for Total Score

If raw score is 30 (such as if a "medium problem" exists with all items), then:

$$
\begin{aligned}
& 100-\left(\frac{20}{40}\right) \times 100 \\
& =100-(0.5 \times 100)=100-50=50 \text { standard score }
\end{aligned}
$$




\section{REFERENCES}

1. Hoff PT, Hogikyan ND. Unilateral vocal fold paralysis. Curr Opin Otolaryngol Head Neck Surg. 1996;4:176-181.

2. Payr. Plastik am Schildknorpel zur Behebung der Folgen einseitiger Stimmbandlahmung. Dtsch Med Wochenschr. 1915; 43:1265.

3. Meurman Y. Operative mediofixation of the vocal cord in complete unilateral paralysis. Arch Otolaryngol. 1952;55: 544-553.

4. Isshiki N, Morita H, Okamura H, Hiramoto M. Thyroplasty as a new phonosurgical technique. Acta Otolaryngol (Stockh). 1974;78:451-457.

5. Isshiki N, Okamura H, Ishikawa T. Thyroplasty type I (lateral compression) for dysphonia due to vocal cord paralysis or atrophy. Acta Otolaryngol (Stockh). 1975;80:465-473.

6. Koufman JA. Laryngoplasty for vocal cord medialization: an alternative to Teflon. Laryngoscope. 1986;96:726-730.

7. Gray SD, Barkmeier J, Jones D, Titze I, Druker D. Vocal evaluation of thyroplastic surgery in the treatment of unilateral vocal fold paralysis. Laryngoscope. 1992;102:415-421.

8. Netterville JL, Stone RE, Lukens ES, Civantos FJ, Ossoff RH. Silastic medialization and arytenoid adduction: the Vanderbilt experience. Ann Otol Rhinol Laryngol. 1993;102:413424.

9. Leder SB, Sasaki CT. Long-term changes in vocal quality following Isskiki thyroplasty type I. Laryngoscope. 1994;104: 275-277.
10. D’Antonio LL, Wigley TL, Zimmerman GJ. Quantitative measures of laryngeal function following teflon injection or thyroplasty type I. Laryngoscope. 1995;105:256-262.

11. Harries ML, Morrison M. Short-term results of laryngeal framework surgery-thyroplasty type I: a pilot study. $J$ Otolaryngol. 1995;24:281-287.

12. Omori K, Kacker A, Slavit D, Blaugrand SM. Quantitative videostroboscopic measurement of glottal gap and vocal function: an analysis of thyroplasty type I. Ann Otol Rhinol Laryngol. 1996;105:280-285.

13. Adams SG, Irish JC, Durkin LC, Wong DLH, Brown DH. Evaluation of vocal function in unilateral vocal fold paralysis following thyroplastic surgery. J Otolaryngol. 1996;25: 165-170.

14. Lu FL, Casiano RR, Lundy DS, Xue JW. Longitudinal evaluation of vocal function after thyroplasty type $I$ in the treatment of unilateral vocal paralysis. Laryngoscope. 1996;106: 573-577.

15. Baba M, Natsugoe S, Shimado M, Nakano S, Noguchi $Y$, Kawachi K, Kusano C, Aikou T. Does hoarseness of voice from recurrent nerve paralysis after esophagectomy for carcinoma influence patient quality of life? J Am Coll Surg. 1999;188:231-236.

16. Gliklich RE, Glovsky RM, Montgomery WW. Validation of a voice outcome survey for unilateral vocal cord paralysis. Otolaryngol Head Neck Surg. 1999;120:153-158.

17. Hogikyan ND, Sethuraman G. Validation of an instrument to measure voice-related quality of life (V-RQOL). $J$ Voice. 1999;13:557-569. 